\title{
Laparoscopic right hemicolectomy: the SICE (Società Italiana di Chirurgia Endoscopica e Nuove Tecnologie) network prospective trial on 1225 cases comparing intra corporeal versus extra corporeal ileo-colic side-to-side anastomosis
}

\author{
Gabriele Anania $^{1,2}$ (D) Ferdinando Agresta ${ }^{3}$. Elena Artioli ${ }^{1,2} \cdot$ Serena Rubino $^{1,2} \cdot$ Giuseppe Resta $^{1,2}$. \\ Nereo Vettoretto ${ }^{4}$ Wanda Luisa Petz ${ }^{5}$. Carlo Bergamini ${ }^{6}$. Alberto Arezzo ${ }^{7}$. Giorgia Valpiani ${ }^{8} \cdot$ Chiara Morotti $^{8}$. \\ Gianfranco Silecchia ${ }^{9} \cdot$ SICE CoDIG (Colon Dx Italian Group)
}

Received: 28 May 2019 / Accepted: 11 November 2019 / Published online: 18 November 2019

(c) The Author(s) 2019

\begin{abstract}
Background While laparoscopic approach for right hemicolectomy (LRH) is considered appropriate for the surgical treatment of both malignant and benign diseases of right colon, there is still debate about how to perform the ileo-colic anastomosis. The ColonDxItalianGroup (CoDIG) was designed as a cohort, observational, prospective, multi-center national study with the aims of evaluating the surgeons' attitude regarding the intracorporeal (ICA) or extra-corporeal (ECA) anastomotic technique and the related surgical outcomes.

Methods One hundred and twenty-five Surgical Units experienced in colorectal and advanced laparoscopic surgery were invited and 85 of them joined the study. Each center was asked not to change its surgical habits. Data about demographic characteristics, surgical technique and postoperative outcomes were collected through the official SICE website database. One thousand two hundred and twenty-five patients were enrolled between March 2018 and September 2018.

Results ICA was performed in $70.4 \%$ of cases, ECA in $29.6 \%$. Isoperistaltic anastomosis was completed in $85.6 \%$, stapled in $87.9 \%$. Hand-sewn enterotomy closure was adopted in $86 \%$. Postoperative complications were reported in $35.4 \%$ for ICA and $50.7 \%$ for ECA; no significant difference was found according to patients' characteristics and technologies used. Median hospital stay was significantly shorter for ICA (7.3 vs. 9 POD). Postoperative pain in patients not prescribed opioids was significantly lower in ICA group.

Conclusions In our survey, a side-to-side isoperistaltic stapled ICA with hand-sewn enterotomy closure is the most frequently adopted technique to perform ileo-colic anastomosis after any indications for elective LRH. According to literature, our study confirmed better short-term outcomes for ICA, with reduction of hospital stay and postoperative pain.
\end{abstract}

Trial registration Clinical trial (Identifier: NCT03934151).

Keywords Right hemicolectomy $\cdot$ Ileo-colic anastomosis $\cdot$ Laparoscopy $\cdot$ Postoperative complications $\cdot$ Intracorporeal anastomosis · Outcomes

While laparoscopic approach for right hemicolectomy (LRH) is considered appropriate for the surgical treatment of both malignant and benign diseases of the right colon, there is still debate about how to perform the ileo-colic anastomosis [1-4].

Gabriele Anania

g.anania@unife.it

Extended author information available on the last page of the article
Over time, different types of ileo-colic anastomosis, such as stapled, hand-sewn and hybrid techniques, have been described and compared. Side-to-side ileo-colic anastomosis is the most frequently used technique by open surgery as well as by minimally invasive approach [5].

Since the intra-corporeal ileo-colic anastomosis (ICA) technique has been proposed [6], the attention was focused on the comparison with the extra-corporeal option. Several cons of the extra-corporeal technique (ECA) have been 
advocated, such as prolonged paralytic ileus, longer postoperative stay, higher risk of wound infection and incisional hernia.

On the other hand, better short-term outcomes related to lower bowel manipulation and stretching, mini Pfannestiel incision used for specimen extraction, have been reported as advantages of the ICA [7-9]. However, ICA is associated to important technical issues: a longer learning curve and operative time as well as higher risk of peritoneal contamination [10].

So far, the only clinical evidence is offered by retrospective low powered studies that indicate faster bowel movements, earlier re-feeding and shorter postoperative hospital stay after LRH with ICA. Prospective comparative multicenter studies comparing perioperative outcomes of ECA versus ICA are still missing [11-27].

The main purpose of this prospective multi-center national study was to evaluate the actual preference of the surgeons concerning intracorporeal and extra-corporeal ileocolic side-to-side anastomosis after LRH as well as to assess the perioperative complications related to the techniques.

\section{Materials and methods}

\section{Study design}

The CoDIG (ColonDxItalianGroup - Italian Right Colon Group) study was designed as a cohort, observational, prospective, multi-center national study comparing ileo-colic side-to-side ECA and ICA techniques after LRH. Patients were recruited from March 2018 to September 2018.

The study was approved and endorsed by SICE (Società Italiana di Chirurgia Endoscopica e Nuove Tecnologie - Italian Society of Endoscopic Surgery and New Technologies).

The Coordinator Center and Promoter of the study is the 1st Department of General Surgery of Ferrara University Hospital.

One hundred and twenty-five Surgical Units, with experience in colorectal laparoscopic surgery were invited and 85 of them (68\%) joined the study. Data were collected using the official SICE website database.

Each center was asked not to change the current practice: the technologies used, the surgical approach, the anastomotic method, the pre- and postoperative management (ERAS protocol and opioid administration included). Patients involved into the study signed an informed consent.

The primary endpoint of the study was to assess the preference of the Italian surgeon (ECA vs. ICA) when performing the ileo-colic side-to-side anastomosis after any indication for LRH.

The secondary endpoint was to compare the postoperative hospital stay, time for re-feeding, time before bowel movement and rate of complications at 30 days after ECA or ICA in LRH.

\section{Population, inclusion/exclusion criteria, data extraction}

Each center was committed to enroll all consecutive cases observed during the study period according to inclusion and exclusion criteria.

Inclusion criteria were age $>18$ years old, elective laparoscopic/robotic right hemicolectomy.

Exclusion criteria were emergency surgery and Body Mass Index $(\mathrm{BMI})>35$.

A section of the official SICE website allowed the online collection of the following data for each patient enrolled:

- Patient's characteristics: gender, age, BMI, major comorbidities (diabetes mellitus, ischemic cardiomyopathy, chronic obstructive arteriopathy, chronic pneumopathy)

- Previous abdominal surgery;

- Indication for surgery: benign or malignant diseases (site of the carcinoma, staging, number of lymph nodes retrieved, number of metastatic lymph nodes)

- Surgical technique: laparoscopy/robotic and the imaging technologies (Full HD, 3D, Indocyanine Green, energy devices) used;

- Type of anastomosis: ECA or ICA, stapled or hand-sewn (single or double layer), isoperistaltic or anti-peristaltic, stapled or hand-sewn enterotomies closure (interrupted or continuous suture);

- Intra-operative complications: hemorrhage, bowel iatrogenic lesions; conversion to open surgery

- Postoperative complications: hemorrhage, anastomotic leakage (method of assessment);

- Postoperative management: ERAS protocol, opioid administration

- Postoperative hospital stay, time for re-feeding, time to return of bowel functions, postoperative pain monitored at scheduled interval $(6,12,24,48 \mathrm{~h})$ through Visual Analog Scale for Pain;

- Hospital readmission within 30 days for surgery related complications.

\section{Statistical analysis}

Categorical data were expressed as total numbers and percentages. Statistical comparisons of categorical variables were assessed using Pearson's $\chi^{2}$ test or Fisher's exact test depending on the minimal expected count in each crosstab. Length of stay was represented with the median and interquartile range $[1 \mathrm{Q}-3 \mathrm{Q}]$ and the Mann-Whitney test was used to analyze the difference between ICA and ECA groups. Unadjusted and adjusted logistic regression were 
used to estimate odds ratios and respective $95 \%$ confidence intervals (95\% CIs). In the multivariate analysis we considered as dependent variable presence of postoperative complications and as covariate: gender, age, presence of previous abdominal surgery, comorbidity, ASA score, operative time and blood loss. Model calibration for multivariate analysis was assessed using the Hosmer-Lemeshow goodness-of-fit test. All analyses were performed using Stata 14.1 SE (Stata Corporation, College Station, Texas, USA). A two-sided $p$ value $<0.05$ was defined as statistically significant.

\section{Results}

One thousand two hundred twenty-five patients were enrolled; 631 (51.5\%) were males and 594 (48.5\%) females. Among all patients, $26.6 \%$ were under 65 years old, and $41 \%$ were older than 75 years. Overweight patients were $37.3 \%$ of all cases, while $13.2 \%$ had a class I obesity.

The indication for elective right hemicolectomy was a benign disease in the $13 \%$ whereas in the residual $87 \%$ the diagnosis was colonic adenocarcinoma, located at the cecum in $39.8 \%$, ascending colon in $37.6 \%$, and transverse colon in $22.7 \%$.

One major comorbidity was present in $22 \%$ of the sample, while $20 \%$ had at least 2 co-morbidities at the time of surgery. Previous abdominal surgery had been performed in $46.7 \%$ of the cases.

The distribution according to ASA score (American Society of Anaesthesiologists) was: 7.6\% ASA I, 49.6\% ASA II, $39.8 \%$ ASA III, 3\% ASA IV (Table 1).

\section{Techniques and technologies}

Surgical procedures were performed in the $92.3 \%$ by laparoscopy, while robotic technique was used in $7.7 \%$ of all cases. Conversion was required in 66 patients (5.4\%), 6 of them performed with robotic technique. Four cases were converted because of intra-operative hemorrhage, 35 cases because of technical difficulties due to overweight, 26 due to massive adhesions and 1 for a iatrogenic intestinal lesion.

In $69.4 \%$ of the procedures these were carried out using a Full HD vision technology, in $25.1 \%$ using 3D vision technology, and in the residual 5.5\% using $4 \mathrm{~K}$ vision technology. Indocyanine Green fluoroangiography was used in $10.4 \%$ of the procedures.

Monopolar/bipolar energy was employed in 5\% of the procedures, radiofrequency energy devices in $43.7 \%$ and ultrasound energy devices in the remaining 51.3\%.

Surgical procedures lasted up to $180 \mathrm{~min}$ in $61.2 \%$ of all cases, 180 to $270 \mathrm{~min}$ in $32.7 \%$, and more than $270 \mathrm{~min}$ in $6.1 \%$. Moreover the duration of surgical procedure is not influenced by the ICA or ECA technique ( $p$ value 0.467 ).
Table 1 Characteristics of patients

\begin{tabular}{|c|c|c|c|}
\hline \multirow[t]{2}{*}{ Type } & \multicolumn{2}{|c|}{ Complications } & \multirow[t]{2}{*}{$n(\%)$} \\
\hline & Yes (489) & No (736) & \\
\hline \multicolumn{4}{|l|}{ Gender } \\
\hline Male & $252(51.5 \%)$ & $379(51.5 \%)$ & $631(51.5)$ \\
\hline Female & $237(48.5 \%)$ & $357(48.5 \%)$ & $594(48.5)$ \\
\hline \multicolumn{4}{|l|}{ Age categories } \\
\hline$<65$ years old & $132(27 \%)$ & $194(26.4 \%)$ & $326(26.6)$ \\
\hline $66-75$ years old & $152(31.1 \%)$ & $240(32.6 \%)$ & $392(32.0)$ \\
\hline$>76$ years old & $205(41.9 \%)$ & $302(41 \%)$ & $507(41.4)$ \\
\hline \multicolumn{4}{|l|}{ BMI } \\
\hline$<30$ & $421(86.1 \%)$ & $642(87.2 \%)$ & $1063(86.8)$ \\
\hline$\geq 30$ & $68(13.9 \%)$ & $94(12.3 \%)$ & $162(13.2)$ \\
\hline \multicolumn{4}{|l|}{ Co-morbidities ${ }^{\mathrm{a}}$} \\
\hline None & $284(58.1 \%)$ & $426(57.9 \%)$ & $710(58.0)$ \\
\hline 1 & $119(24.3 \%)$ & $153(20.8 \%)$ & $272(22.2)$ \\
\hline$\geq 2$ & $86(17.6 \%)$ & $157(21.3 \%)$ & $243(19.8)$ \\
\hline \multicolumn{4}{|l|}{ ASA score } \\
\hline 1 & $43(8.8 \%)$ & $50(6.8 \%)$ & $93(7.6)$ \\
\hline 2 & $236(48.2 \%)$ & $371(50.4 \%)$ & 607 (49.6) \\
\hline 3 & $196(40.1 \%)$ & $292(39.7 \%)$ & $488(39.8)$ \\
\hline 4 & $14(2.9 \%)$ & $23(3.1 \%)$ & $37(3)$ \\
\hline \multicolumn{4}{|c|}{ Previous abdominal surgery } \\
\hline None & $274(56 \%)$ & $379(51.5 \%)$ & $653(53.3)$ \\
\hline 1 & $215(44 \%)$ & $357(48.5 \%)$ & $572(46.7)$ \\
\hline \multicolumn{4}{|l|}{ Pathology } \\
\hline Benign & $58(11.9 \%)$ & $99(13.4 \%)$ & $157(12.8)$ \\
\hline Malignant & $431(88.1 \%)$ & $637(86.6 \%)$ & $1068(87.2)$ \\
\hline \multicolumn{4}{|c|}{ Location of the disease } \\
\hline Cecum & $207(42.3 \%)$ & $280(38 \%)$ & 487 (39.8) \\
\hline Ascending colon & $183(37.4 \%)$ & $277(37.7 \%)$ & 460 (37.6) \\
\hline Trasverse colon & $99(20.3 \%)$ & $179(24.3 \%)$ & 278 (22.7) \\
\hline Total & & & $1225(100)$ \\
\hline
\end{tabular}

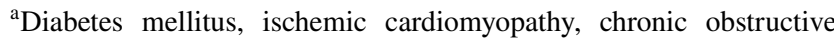
arteriopathy, chronic pneumopathy

Intra-operative blood loss was $<200 \mathrm{ml}$ in $94.3 \%$, among 201 and $299 \mathrm{ml}$ in $0.4 \%$ and $>$ than $300 \mathrm{ml}$ in 5.3 of all cases. Blood transfusions during surgery were administered in $6.4 \%$ of the procedures (Table 2).

\section{Anastomosis}

ICA was performed in 862 cases (70.4\%), ECA in 363 cases $(29.6 \%)$.

In ICA group, isoperistaltic anastomosis was completed in $88.4 \%$ of patients, stapled in $97 \%$. Concerning enterotomies closure, a manual suture was adopted in $95.7 \%$ of the cases (79\% double layer, $17.2 \%$ single layer, with continuous suture in $88.4 \%$ ). 
Table 2 Descriptive analysis of technologies used and intervention variables

\begin{tabular}{llll}
\hline Variables & Type & $n(\%)$ & \\
\hline Technique used & Laparoscopic & $1131(92.3 \%)$ & \\
& Robotic & $94(7.7 \%)$ & \\
Visual technology & Full HD & $850(69.4 \%)$ & \\
& 3D & $308(25.1 \%)$ & \\
& $4 \mathrm{~K}$ & $67(5.5 \%)$ & \\
Indocyanin green & Yes & $127(10.4 \%)$ & \\
& No & $1098(89.6 \%)$ & \\
Energy devices & Radiofrequency & $536(43.7 \%)$ & \\
& Ultrasound & $628(51.3 \%)$ & \\
Length of interven- & Minutes & ICA (862) & ECA (363) \\
tion & $90-180$ & $519(60.2 \%)$ & $231(63.6 \%)$ \\
& $180-270$ & $287(33.3 \%)$ & $113(31.1 \%)$ \\
& $>270$ & $56(6.5 \%)$ & $19(5.2 \%)$ \\
Blood loss (ml) & $0-200$ & $1155(94.3 \%)$ & \\
& $201-299$ & $5(0.4 \%)$ & \\
Lymph nodes & $\geq 300$ & $65(5.3 \%)$ & \\
removed & $<12$ & $79(6.4 \%)$ & \\
& $\geq 12$ & $981(80.1 \%)$ & \\
& Missing & $165(13.5 \%)$ & \\
& & & \\
& & $61(5 \%)$ & \\
& & & \\
& & &
\end{tabular}

Table 3 Characteristic of anastomosis

\begin{tabular}{llll}
\hline Variables & Type & ICA (\%) & ECA (\%) \\
\hline Direction & Anisoperistaltic & $100(11.6)$ & $77(21.2)$ \\
Side-to-side & Isoperistaltic & $762(88.4)$ & $286(78.8)$ \\
& Manual & $26(3)$ & $122(33.6)$ \\
Enterotomy closure & Mechanical & $836(97)$ & $241(66.4)$ \\
& Manual & $825(95.7)$ & $228(62.8)$ \\
Enterotomy manual & Mechanical & $37(4.3)$ & $135(37.2)$ \\
closure & Single layer & $148(17.2)$ & $62(17.1)$ \\
& Double layer & $681(79)$ & $184(50.7)$ \\
& missing & $33(3.8)$ & $117(32.2)$ \\
& Continuous suture & $762(88.4)$ & $183(50.4)$ \\
& Interrupted suture & $36(4.2)$ & $58(16)$ \\
Mesocolon closure & Missing & $64(7.4)$ & $122(33.6)$ \\
& No & $378(43.8)$ & $140(38.6)$ \\
& Yes & $484(56.2)$ & $223(61.4)$ \\
\hline
\end{tabular}

In ECA group, isoperistaltic anastomosis was performed in $78.8 \%$, stapled in $66.4 \%$. Manual enterotomy closure was adopted in $62.8 \%$ (50.7\% double layer, $17.1 \%$ single layer, with continuous suture in 50.4\%) (Table 3 ).

\section{Intra-operative complications}

Intra-operative complications were reported in $20(1.6 \%)$ patients; 4 of which $(1.3 \%)$ were intraperitoneal hemorrhages and $1(0.3 \%)$ iatrogenic small bowel lesion.

\section{Postoperative outcomes}

ERAS protocol was applied to 655 patients (53.5\%), of which 537 belonging to ICA group (62.3\% of ICA group) and 118 belonging to ECA group (32.5\% of ECA group).

In $22.8 \%$ of patients gas passed during the first postoperative day (POD), in $66.4 \%$ between the second and the third POD, in $10.8 \%$ after the fourth POD.

A liquid diet was administered during the first POD to $11.1 \%$ of the patients, to $61.4 \%$ between the first and the second POD, to $19.4 \%$ between third and fourth POD and to $8.1 \%$ after the fourth POD.

A solid diet was restored on the same day of the operation in $0.8 \%$ of patients, between the first and the second POD in $37.6 \%$, between the third and the fourth POD in $41.1 \%$ and after the fourth POD in $20.6 \%$.

Patients were discharged within the fourth POD in $16.4 \%$ of cases, in $58.8 \%$ between the fifth and the eighth POD and after the eighth POD in $24.8 \%$ (Table 4).

\section{Postoperative complications}

Postoperative complications have been recorded in 489 patients (39.9\%); no complications have occurred in 736 patients $(60.1 \%)$. Main complications were anastomotic bleeding (4\%), anastomotic leakage (2.2\%), bowel obstruction (1.7\%), intra-abdominal abscess (1.8\%), wound infection (4.3\%) [28]. Minor complications included in grade 1 and 2 of Clavien-Dindo scale [29] were reported in 25.9\% of all patients (Table 5).

Twenty-nine patients (2\%) were readmitted in hospital within 30 days, mainly for bowel obstruction and wound infection. Four required further surgery: 1 for anastomotic leakage and 3 for bowel obstruction (Table 6).

Univariate analysis was performed for risk factors for postoperative complications on the sample stratified by clinical, surgical and personal characteristics. No statistically significant difference was found according to patient's gender, age, BMI, co-morbidities, ASA score, previous abdominal surgery, benign or malignant disease and location. The likelihood of developing postoperative complications did not depend, in a statistically significant way, on the use of laparoscopic/robotic technique as well as the imaging technology and the type of energy source used. The use of laparoscopic/ robotic technique as well as the imaging technology and the 
Table 4 Postoperative recovery differences between groups

\begin{tabular}{|c|c|c|c|c|}
\hline Variables & $n=1225$ & ICA $(n=862)$ & $\operatorname{ECA}(n=363)$ & $p$ value \\
\hline Time to flatus passage (days) & & & & $<0.0001$ \\
\hline 1 & $279(22.8 \%)$ & $239(27.7 \%)$ & $40(11 \%)$ & \\
\hline $2-3$ & $814(66.4 \%)$ & $554(64.3 \%)$ & $260(71.6 \%)$ & \\
\hline$\geq 4$ & $132(10.8 \%)$ & $69(8 \%)$ & $63(17.4 \%)$ & \\
\hline Time to bowel movement (days) & & & & $<0.0001$ \\
\hline $1-2$ & $263(21.5 \%)$ & $223(25.9 \%)$ & $40(11 \%)$ & \\
\hline $3-5$ & $851(69.5 \%)$ & $572(66.3 \%)$ & $279(76.9 \%)$ & \\
\hline$>5$ & $111(9.1 \%)$ & $67(7.8 \%)$ & $44(12.1 \%)$ & \\
\hline Resumption of liquid diet (days) & & & & $<0.0001$ \\
\hline 0 & $136(11.1 \%)$ & $128(14.9 \%)$ & $8(2.2 \%)$ & \\
\hline $1-2$ & $752(61.4 \%)$ & $577(66.9 \%)$ & $175(48.2 \%)$ & \\
\hline $3-4$ & $238(19.4 \%)$ & $113(12.1 \%)$ & $125(34.4 \%)$ & \\
\hline$>5$ & $99(8.1 \%)$ & $44(5.1 \%)$ & $55(15.2 \%)$ & \\
\hline Resumption of solid diet (days) & & & & $<0.0001$ \\
\hline 0 & $10(0.8 \%)$ & $9(1 \%)$ & $1(0.3 \%)$ & \\
\hline $1-2$ & $460(37.6 \%)$ & $396(45.9 \%)$ & $64(17.6 \%)$ & \\
\hline $3-4$ & $503(41.1 \%)$ & $332(38.5 \%)$ & $171(47.1 \%)$ & \\
\hline$\geq 5$ & $252(20.6 \%)$ & $125(14.5 \%)$ & $127(35 \%)$ & \\
\hline Hospital stay (days) & & & & $<0.0001$ \\
\hline $0-4$ & $201(16.4 \%)$ & $190(22.1 \%)$ & $11(3 \%)$ & \\
\hline $5-8$ & $720(58.8 \%)$ & $503(58.3 \%)$ & $217(59.8 \%)$ & \\
\hline$\geq 9$ & $304(24.8 \%)$ & $169(19.6 \%)$ & $135(37.2 \%)$ & \\
\hline Length of stay (days) & & & & $<0.0001$ \\
\hline (Median) [QR] & $570(46.5 \%)$ & $6[5-8]$ & $8[7-10]$ & \\
\hline ERAS protocol & & & & $<0.0001$ \\
\hline Yes & $655(53.5 \%)$ & $537(62.3 \%)$ & $118(32.5 \%)$ & \\
\hline No & $570(46.5 \%)$ & $325(37.7 \%)$ & $245(67.5 \%)$ & \\
\hline
\end{tabular}

Table 5 Postoperative complications

\begin{tabular}{llllc}
\hline Complications & ECA (\%) & ICA (\%) & N (\%) & $p$ value \\
\hline Wound infection & $23(6.3)$ & $30(3.5)$ & $53(4.3)$ & 0.021 \\
Bleeding & $12(3.3)$ & $37(4.3)$ & $49(4)$ & 0.421 \\
Leakage & $6(1.6)$ & $21(2.4)$ & $27(2.2)$ & 0.394 \\
Abdominal abscess & $4(1.1)$ & $18(2.1)$ & $22(1.8)$ & 0.235 \\
Bowel obstruction & $5(1.38)$ & $16(1.86)$ & $21(1.7)$ & 0.556 \\
Clavien-Dindo 1-2 & $134(36.9)$ & $183(21.2)$ & $317(25.9)$ & $<0.0001$ \\
None & $179(49.3)$ & $557(64.6)$ & $736(60.1)$ & $<0.0001$ \\
Total & $363(100)$ & $862(100)$ & 1225 & \\
\hline
\end{tabular}

type of energy source used did not influence statistically the likelihood of developing postoperative complications (Table 7).

The ileo-colic side-to-side anastomosis direction (anisoperistaltic or isoperistaltic), technique (stapled or hand-sewn) and the method of enterotomy closure did not influence the anastomotic bleeding and leakage (Table 8).

Adjusted logistic regression analysis showed that age, comorbidities, previous abdominal surgery, gender, ASA score,
Table 6 Reasons for readmission within 30 days

\begin{tabular}{llll}
\hline 30-days readmission causes & ECA $(\%)$ & ICA $(\%)$ & $N(\%)$ \\
\hline Intestinal obstruction & $1(11.1)$ & $9(45)$ & $10(34.5)$ \\
Anemia and wound infection & $5(55.6)$ & $3(15)$ & $8(27.6)$ \\
Pneumonia & $1(11.1)$ & $1(5)$ & $2(6.9)$ \\
Anemia and rectal bleeding & $1(11.1)$ & $1(5)$ & $2(6.9)$ \\
Abdominal pain and fever & $0(0)$ & $1(5)$ & $1(3.4)$ \\
Nausea and diarrhea & $1(11.1)$ & $2(10)$ & $3(10.4)$ \\
Blood effusion & $0(0)$ & $1(5)$ & $1(3.4)$ \\
Intra-abdominal abscess & $0(0)$ & $1(5)$ & $1(3.4)$ \\
Anastomotic leakage & $0(0)$ & $1(5)$ & $1(3.4)$ \\
Total & $9(100)$ & $20(100)$ & $29(100)$ \\
\hline
\end{tabular}

operative time and blood loss did not influenced the likelihood of developing postoperative complications (Table 7).

To analyse the length of intervention in relation to the surgical technique, the sample was purified from conversions and intra-operative complications which would influence the results by increasing the operating time. The intervention lasted $90-180 \mathrm{~min}$ in $60.2 \%$ of ICA and $68.1 \%$ of 
Table 7 Univariate and multivariate analysis for risk factors for postoperative complications

\begin{tabular}{|c|c|c|c|c|c|c|c|}
\hline \multirow[t]{3}{*}{ Patient characteristics } & \multirow[t]{3}{*}{ Reference category } & \multicolumn{3}{|c|}{ Unadjusted OR } & \multicolumn{3}{|c|}{ Adjusted OR } \\
\hline & & OR & $95 \%$ & CI & OR & $95 \%$ & $\mathrm{CI}$ \\
\hline & & & Lower & Upper & & Lower & Upper \\
\hline Male & Female & 1.0157 & 0.796 & 1.259 & 0.991 & 0.783 & 1.317 \\
\hline Age categories & $<65$ & & & & & & \\
\hline $66-75$ & & 0.9308 & 0.689 & 1.256 & 0.965 & 0.707 & 1.317 \\
\hline$>76$ & & 0.9976 & 0.751 & 1.324 & 1.040 & 0.761 & 1.421 \\
\hline Comorbidity & None & & & & & & \\
\hline 1 & & 1.1666 & 0.879 & 1.547 & 1.1944 & 0.873 & 1.632 \\
\hline More than 1 & & 0.8216 & 0.607 & 1.112 & 0.8368 & 0.582 & 1.202 \\
\hline ASA score & 1 & & & & & & \\
\hline 2 & & 0.7396 & 0.476 & 1.147 & 0.7446 & 0.472 & 1.173 \\
\hline 3 & & 0.7805 & 0.499 & 1.219 & 0.7911 & 0.475 & 1.315 \\
\hline 4 & & 0.7077 & 0.324 & 1.543 & 0.7781 & 0.335 & 1.803 \\
\hline Previous abdominal surgery & None & & & & & & \\
\hline Yes & & 0.8330 & 0.662 & 1.048 & 0.8470 & 0.669 & 1.072 \\
\hline Operative time (minutes) & $90-180$ & & & & & & \\
\hline $180-270$ & & 0.851 & 0.663 & 1.091 & 0.8455 & 0.658 & 1.093 \\
\hline$>270$ & & 0.744 & 0.453 & 1.224 & 0.7596 & 0.454 & 1.237 \\
\hline Blood losses (ml) & $0-200$ & & & & & & \\
\hline $201-299$ & & 1 & 0.166 & 6.007 & 1.209 & 0.198 & 7.375 \\
\hline$\geq 300$ & & 0.937 & 0.561 & 1.566 & 0.9408 & 0.559 & 1.582 \\
\hline BMI & $<30$ & & & & & & \\
\hline$\geq 30$ & & 1.1031 & 0.788 & 1.542 & & & \\
\hline Pathology & Benign & & & & & & \\
\hline Malignant & & 1.1549 & 0.816 & 1.632 & & & \\
\hline Location & Cecum & & & & & & \\
\hline Ascending colon & & 0.8936 & 0.689 & 1.157 & & & \\
\hline Trasversum & & 0.7481 & 0.551 & 1.014 & & & \\
\hline Technique used & Laparoscopic-videoassistited & & & & & & \\
\hline Robotic & & 0.8419 & 0.543 & 1.303 & & & \\
\hline Technology & FullHD & & & & & & \\
\hline $3 \mathrm{D}$ & & 0.9684 & 0.742 & 1.263 & & & \\
\hline $4 \mathrm{~K}$ & & 0.6198 & 0.360 & 1.064 & & & \\
\hline Indocyanine Green & Yes & & & & & & \\
\hline No & & 1.1909 & 0.813 & 1.743 & & & \\
\hline Dissection technology & Radiofrequency & & & & & & \\
\hline Ultrasounds & & 1.174 & 0.927 & 1.486 & & & \\
\hline Monopolar/bipolar & & 1.064 & 0.618 & 1.830 & & & \\
\hline No. of lymph nodes harvested & $<12$ & & & & & & \\
\hline$\geq 12$ & & 1.043 & 0.652 & 1.668 & & & \\
\hline
\end{tabular}

ECA, it lasted $181-270 \mathrm{~min}$ in $33.3 \%$ of ICA and $27.9 \%$ of ECA, more than 270 min in $6.5 \%$ of ICA and $4 \%$ of ECA. Operating time in patients receiving an ICA resulted significant longer than in patients receiving an ECA $(p=0.037)$ (Table 9).

Significant difference was found between ICA and ECA groups about postoperative complications. ICA group showed a lower rate of total complications and of Clavien-Dindo grade I-II, whereas higher rate of wound infections was associated to ECA technique. No differences were found among bleeding, leakage, abdominal abscess and bowel obstruction (Table 5).

Statistically significant differences have been found comparing ICA and ECA groups in relation to postoperative 
Table 8 Association between surgical variables and anastomotic complications

\begin{tabular}{|c|c|c|c|c|c|c|}
\hline \multirow[t]{2}{*}{ Variables } & \multicolumn{3}{|l|}{ Bleeding } & \multicolumn{3}{|l|}{ Leakage } \\
\hline & Bleeding $(n=49)$ & No bleeding $(n=1176)$ & $p$ value & Leakage $(n=27)$ & No leakage $(n=1198)$ & $p$ value \\
\hline \multicolumn{7}{|l|}{ Anastomosis } \\
\hline Intracorporeal & $37(75.5 \%)$ & $825(70.2 \%)$ & \multirow[t]{2}{*}{0.421} & $21(77.8 \%)$ & $841(70.2 \%)$ & \multirow[t]{2}{*}{0.394} \\
\hline Extra-corporeal & $12(24.5 \%)$ & $351(29.8 \%)$ & & $6(22.2 \%)$ & $357(29.8 \%)$ & \\
\hline \multicolumn{7}{|c|}{ Anastomosis direction } \\
\hline Anisoperistaltic & $4(8.2 \%)$ & $173(14.7 \%)$ & \multirow[t]{2}{*}{0.202} & $6(22.2 \%)$ & $171(14.3 \%)$ & \multirow[t]{2}{*}{0.264} \\
\hline Isoperistaltic & $45(91.8 \%)$ & $1003(85.3 \%)$ & & $21(77.8 \%)$ & $1027(85.7 \%)$ & \\
\hline \multicolumn{7}{|c|}{ Anastomosis confectioning } \\
\hline Manual & $3(6.1 \%)$ & $145(12.3 \%)$ & \multirow[t]{2}{*}{0.191} & $3(11.1 \%)$ & $145(12.1 \%)$ & \multirow[t]{2}{*}{0.876} \\
\hline Mechanical & $46(93.9 \%)$ & $1031(87.7 \%)$ & & $24(88.9 \%)$ & $1053(87.9 \%)$ & \\
\hline \multicolumn{7}{|l|}{ Enterotomy closure } \\
\hline Manual & $43(87.8 \%)$ & $1010(85.9 \%)$ & \multirow[t]{2}{*}{0.712} & $25(92.6 \%)$ & $1028(85.8 \%)$ & \multirow[t]{2}{*}{0.316} \\
\hline Mechanical & $6(12.2 \%)$ & $166(14.1 \%)$ & & $2(7.4 \%)$ & $170(14.2 \%)$ & \\
\hline \multicolumn{7}{|l|}{ Mesocolon closure } \\
\hline No & $28(57.1 \%)$ & $490(41.7 \%)$ & \multirow[t]{3}{*}{0.082} & $10(37 \%)$ & $508(42.4 \%)$ & \multirow[t]{3}{*}{0.287} \\
\hline Yes, continuous & $11(22.5 \%)$ & $412(35 \%)$ & & $13(48.2 \%)$ & $410(34.2 \%)$ & \\
\hline Yes, interrupted & $10(20.4 \%)$ & $274(23.3 \%)$ & & $4(14.8 \%)$ & $280(23.4 \%)$ & \\
\hline \multicolumn{7}{|c|}{ Enterotomy manual closure ${ }^{\mathrm{a}}$} \\
\hline Single layer & $8(19 \%)$ & $202(19.5 \%)$ & \multirow[t]{2}{*}{0.935} & $6(24 \%)$ & $204(19.4 \%)$ & \multirow[t]{2}{*}{0.569} \\
\hline Double layer & $34(81 \%)$ & $831(80.5 \%)$ & & $19(76 \%)$ & $846(80.6 \%)$ & \\
\hline \multicolumn{7}{|c|}{ Enterotomy hand-sewn suture ${ }^{\mathrm{b}}$} \\
\hline Continuous & $39(95.1 \%)$ & $906(90.8 \%)$ & \multirow[t]{2}{*}{0.342} & $21(91.3 \%)$ & $924(90.9 \%)$ & \multirow[t]{2}{*}{0.953} \\
\hline Interrupted & $2(4.8 \%)$ & $92(9.2 \%)$ & & $2(8.7 \%)$ & $92(9.1 \%)$ & \\
\hline
\end{tabular}

${ }^{\text {a } 9}$ missing values

${ }^{b} 12$ missing values

Table 9 Differences in length of intervention between groups

\begin{tabular}{llll}
\hline Variables & \multicolumn{2}{c}{ Anastomosis } & \multirow{2}{*}{$p$ value } \\
\cline { 2 - 3 } & $\operatorname{ICA}(846)^{\mathrm{a}}$ & $\mathrm{ECA}(298)^{\mathrm{a}}$ & \\
\hline Lenght of intervention & & & \multirow{2}{*}{0.037} \\
$90-180 \mathrm{~min}$ & $509(60.2 \%)$ & $203(68.1 \%)$ & \\
$181-270 \mathrm{~min}$ & $282(33.3 \%)$ & $83(27.9 \%)$ & \\
$>270 \mathrm{~min}$ & $55(6.5 \%)$ & $12(4 \%)$ & \\
\hline
\end{tabular}

${ }^{a}$ Sample purified from conversions and intra-operative complications

outcome. Patients in ICA group showed a shorter time of bowel function recovery, of resumption of liquid and solid diet, and of median length of stay (6 vs. $8, p<0.0001$ ) (Table 4).

Considering that ICA group had fewer complications than ECA group, we analyzed the postoperative outcome excluding patients converted to laparotomy, who had intraoperative and postoperative complications, and who were admitted into the intensive care unit in order to rule out potential confounding factors. Even in this sample, ICA is significantly associated with a better short-term outcome $(p<0.0001)$ (Table 10).
Because of the great difference among the percentage of the ICA vs ECA cohorts receiving ERAS, a separated analysis of postoperative outcomes in the two groups ERAS and no-ERAS was carried out. In the group following ERAS protocol, all variables regarding postoperative outcome were significantly better in patients chosen for ICA. Analyzing the no-ERAS cohorts, patients chosen for ICA had faster resumption of liquid and solid diet, with shorter hospital stay even if the median LOS was equal to ECA group. No statistical significance had been found regarding the resumption of bowel movement which, anyway, resulted earlier in ICA group (Table 11).

Postoperative pain within $48 \mathrm{~h}$ after surgery was similar in the two groups (Fig. 1), but the subgroup analysis of those patients in whom opioids were not prescribed demonstrated a significant reduction of the mean reported pain in the ICA group except for the detection of pain within $6 \mathrm{~h}$ (Table 12). 
Table 10 Postoperative recovery differences between groups

\begin{tabular}{llll}
\hline Variables & ICA $(n=486)^{\mathrm{a}}$ & ECA $(n=130)^{\mathrm{a}}$ & $p$ value \\
\hline Time to flatus passage (days) & & & $<0.0001$ \\
1 & $156(32.1 \%)$ & $16(12.3 \%)$ & \\
$2-3$ & $311(64 \%)$ & $101(77.7 \%)$ & 0.013 \\
$\geq 4$ & $19(3.9 \%)$ & $13(10 \%)$ & \\
Time to bowel movement (days) & & & \\
$1-2$ & $151(31.1 \%)$ & $24(18.5 \%)$ & $<0.0001$ \\
$3-5$ & $319(65.6 \%)$ & $102(78.5 \%)$ & \\
$>5$ & $16(3.3 \%)$ & $4(3 \%)$ & $<0.0001$ \\
Resumption of liquid diet (days) & & $1(0.7 \%)$ & \\
0 & $81(16.7 \%)$ & $81(62.3 \%)$ & \\
$1-2$ & $352(72.4 \%)$ & $46(35.4 \%)$ & \\
$3-4$ & $38(7.8 \%)$ & $2(1.6 \%)$ & \\
$>5$ & $15(3.1 \%)$ & 0.0001 \\
Resumption of solid diet (days) & & $7(0 \%)$ & \\
0 & $8(1.6 \%)$ & $29(22.3 \%)$ & \\
$1-2$ & $251(51.6 \%)$ & $87(66.9 \%)$ & \\
$3-4$ & $190(39.1 \%)$ & $6(4.6 \%)$ & \\
$\geq 5$ & $37(7.6 \%)$ & $37(28.5 \%)$ & \\
Length of stay (days) & $152(31.3 \%)$ & $295(60.7 \%)$ & \\
$0-4$ & $39(8 \%)$ & & \\
$5-8$ & $5[47]$ & & \\
$\geq 9$ & & & \\
Length of stay (days) & & & \\
(median) [QR] & & & \\
\hline a sample purified from conversions, intra and & postoperative complications, admission in intensive care unit
\end{tabular}

\section{Discussion}

Nowadays, minimally invasive surgery is accepted as standard approach for benign and malignant colon diseases on the basis of proven short and long-term outcomes.

In 2003 the totally laparoscopic side-to-side ileo-colic stapled anastomosis was proposed after elective LRH [6] and, today, it is still considered a challenging procedure which requires proper learning curve. In facts, with ICA the rate of intraperitoneal contamination is higher, especially in patients without mechanical bowel preparation before surgery [10, 21-23].

ICA was indicated first in case of obesity, to avoid the bigger incision necessary to extract the ileo-colic specimen and the need of mesentery traction during ECA confectioning. Furthermore, it was reported that ECA was associated with a higher rate of wound infection particularly in obese patients [7-9, 13, 24, 30].

Over the years, retrospective monocenter studies and a few meta-analysis comparing ICA vs ECA, showed best short terms outcomes in patients undergoing ICA. Just a single study showed a lower rate of anastomotic leakage in case of ICA [11]. A recent meta-analysis confirms a lower perioperative morbidity in ICA group [20]. Nonetheless, no prospective multi-center observational trial has compared totally intracorporeal vs extra-corporeal side-to-side ileocolic anastomosis technique to define advantages and disadvantages and to offer recommendations to surgeons and stakeholders [23, 24, 31, 32].

In order to support the clinical practice of the members and to guarantee the best patients care, the Scientific Committee of SICE promoted the present observational prospective cohort study to collect data and frame the current surgical practice in Italy. 85 accredited surgical units with at least two active SICE members in each center were involved [33].

During the observational period, the participating surgeons (210 accredited laparoscopic surgeons) recruited a large cohort of 1225 consecutive cases respecting the inclusion criteria. According to our knowledge, the present observational prospective study has the largest sample of elective right hemicolectomy performed with mini-invasive technique that compares ICA versus ECA.

The present study involved high volume colorectal units with expertise in colorectal and advanced laparoscopic surgery, therefore surgeons were probably predisposed to perform more complex surgical procedures, like ICA during LRH. This represents a possible bias for the following results. 
Table 11 Postoperative recovery in ERAS and no-ERAS groups in relation to anastomotic technique

\begin{tabular}{|c|c|c|c|c|c|c|}
\hline \multirow[t]{2}{*}{ Variables } & \multicolumn{3}{|l|}{ ERAS (655) } & \multicolumn{3}{|c|}{ no-ERAS (570) } \\
\hline & ICA (537) & ECA (118) & $p$ value & ICA (325) & ECA (245) & $p$ value \\
\hline Time to flatus passage (days) & & & $<0.001$ & & & 0.490 \\
\hline 1 & $208(38.7 \%)$ & $13(11 \%)$ & & $31(9.5 \%)$ & $27(11 \%)$ & \\
\hline $2-3$ & $310(57.7 \%)$ & $87(73.7 \%)$ & & $244(75.1 \%)$ & $173(70.6 \%)$ & \\
\hline$>4$ & $19(3.6 \%)$ & $18(15.3 \%)$ & & $50(15.4 \%)$ & $45(18.4 \%)$ & \\
\hline Time to bowel movement (days) & & & $<0.001$ & & & 0.110 \\
\hline $1-2$ & $170(31.7 \%)$ & $15(12.7 \%)$ & & $53(16.3 \%)$ & $25(10.2 \%)$ & \\
\hline $3-5$ & $345(64.2 \%)$ & $95(80.5 \%)$ & & $227(69.8 \%)$ & $184(75.1 \%)$ & \\
\hline$>5$ & $22(4.1 \%)$ & $8(6.8 \%)$ & & $45(13.9 \%)$ & $36(14.7 \%)$ & \\
\hline Resumption of liquid diet (days) & & & $<0.001$ & & & $<0.001$ \\
\hline 0 & $128(23.8 \%)$ & $7(5.9 \%)$ & & $0(0 \%)$ & $1(0.4 \%)$ & \\
\hline $1-2$ & $379(70.6 \%)$ & $71(60.2 \%)$ & & $198(60.9 \%)$ & $104(42.4 \%)$ & \\
\hline $3-4$ & $23(4.3 \%)$ & $31(26.3 \%)$ & & $90(27.7 \%)$ & $94(38.4 \%)$ & \\
\hline$>5$ & $7(1.3 \%)$ & $9(7.6 \%)$ & & $37(11.4 \%)$ & $46(18.8 \%)$ & \\
\hline Resumption of solid diet (days) & & & $<0.001$ & & & $<0.001$ \\
\hline 0 & $9(1.7 \%)$ & $0(0 \%)$ & & $0(0 \%)$ & $1(0.4 \%)$ & \\
\hline $1-2$ & $340(63.3 \%)$ & $46(39 \%)$ & & $56(17.2 \%)$ & $18(7.3 \%)$ & \\
\hline $3-4$ & $158(29.4 \%)$ & $44(37.3 \%)$ & & $174(53.5 \%)$ & $127(51.8 \%)$ & \\
\hline$>5$ & $30(5.6 \%)$ & $28(23.7 \%)$ & & $95(29.3 \%)$ & $99(40.5 \%)$ & \\
\hline Hospital stay (days) & & & $<0.001$ & & & 0.040 \\
\hline $0-4$ & $179(33.3 \%)$ & $9(7.6 \%)$ & & $11(3.4 \%)$ & $2(0.8 \%)$ & \\
\hline $5-8$ & $306(57 \%)$ & $80(67.8 \%)$ & & $197(60.6 \%)$ & $137(55.9 \%)$ & \\
\hline$>9$ & $52(9.7 \%)$ & $29(24.6 \%)$ & & $117(36 \%)$ & $106(43.3 \%)$ & \\
\hline Leight of stay (days) & & & $<0.001$ & & & 0.0163 \\
\hline (median) $[\mathrm{QR}]$ & 5 [4-7] & 7 [6-8] & & 8 [6-10] & 8 [7-10] & \\
\hline
\end{tabular}

Fig. 1 Mean pain severity divided by group within $48 \mathrm{~h}$ post-surgery

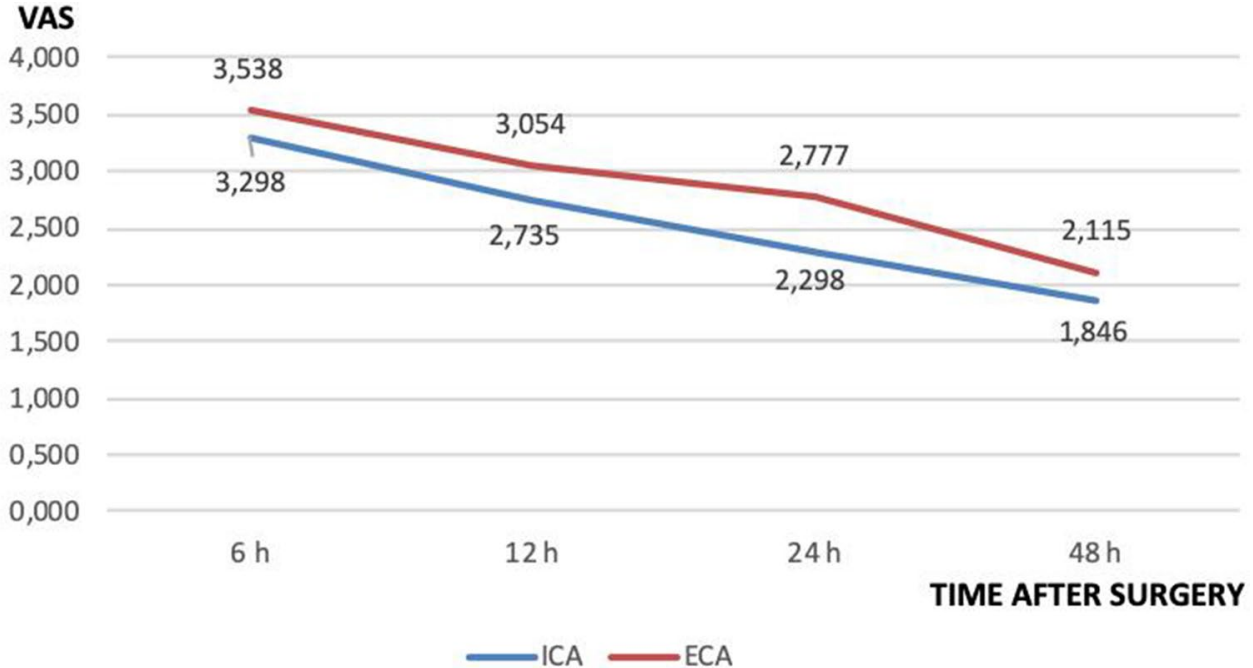

The results of the survey demonstrated that the large majority of the Italian surgeons involved performs a sideto-side isoperistaltic totally intracorporeal stapled anastomosis after any indications for elective laparoscopic right hemicolectomy with hand-sewn closure of the enterotomies (preferred double layer with continuous suture). The majority of the surgeons used Full HD vision technology; only in the $7.5 \%$ of the cases a robotic setting was used.

The length of intervention resulted higher in ICA group, consistent with the longer time necessary to perform the anastomosis with the completely intracorporeal technique. 
Table 12 Distribution of VAS scale (mean SD) VAS by intervals post-surgery

\begin{tabular}{|c|c|c|c|c|c|c|}
\hline \multirow{3}{*}{$\begin{array}{l}\text { Opiod administration } \\
\text { Time classes (h) }\end{array}$} & \multicolumn{2}{|l|}{ Yes } & \multirow[t]{3}{*}{$p$ value } & \multicolumn{2}{|l|}{ No } & \multirow[t]{3}{*}{$p$ value } \\
\hline & \multicolumn{2}{|c|}{ Type of anastomosis } & & \multicolumn{2}{|c|}{ Type of anastomosis } & \\
\hline & $\operatorname{ICA}(n=213)$ & ECA $(n 74)$ & & $\operatorname{ICA}(n=273)$ & ECA $(n 56)$ & \\
\hline 6 & $3.357(1.921)$ & $3.445(2.088)$ & 0.7371 & $3.253(1.734)$ & 3.661 (1.947) & 0.1174 \\
\hline 12 & $2.943(1.701)$ & 2.919 (1.907) & 0.9169 & $2.571(1.402)$ & $3.232(1.737)$ & 0.0023 \\
\hline 24 & $2.455(1.579)$ & 2.689 (1.719) & 0.2847 & $2.176(1.311)$ & $2.893(1.626)$ & 0.0004 \\
\hline 48 & $2.098(1.468)$ & $1.851(1.201)$ & 0.1922 & $1.648(0.936)$ & $2.464(1.584)$ & $<0.0001$ \\
\hline
\end{tabular}

The analysis of the characteristics of patients stratified according to the anastomotic technique (ICA vs. ECA) allowed to select two homogeneous groups for the statistical analysis of perioperative complications.

Our sample comprised a large portion of elderly patients ( $>65$ years old) with co-morbidities and previous abdominal surgery, but none of those parameters influenced the postoperative complications rate in subgroups multivariate analysis in relation to the anastomotic technique (ICA vs. ECA). The multivariate analysis did not identify any predictive factor for anastomosis complications. This finding is in contrast with previous reports [34-36] and could be related to a possible bias of this study involving high volume colorectal units with expertise in colorectal surgery.

We observed that the total number of complications as well as minor complications (Clavien-Dindo grade I-II) were influenced by the anastomotic technique, with a lower rate in ICA group. On the contrary, wound infection resulted higher in ECA group and it also represented the most frequent complication. For other complications like, in order of frequency, anastomotic bleeding and leakage, no statistical difference had been found between the two groups.

Hospital readmission rate within 30 days was low (2\%).

In accordance to what reported in literature [4, 7, 24, 31], we observed better short term outcomes and a significant decrease of the hospital stay in ICA group than ECA, both in the total sample and, as further confirmation, in a sample purified from any complications.

ERAS protocol could be a confounding factor for these results because, even if the population looks homogeneous with $53.3 \%$ following ERAS protocol and $46.5 \%$ not, actually it is more applied in ICA group than ECA (62.3\% vs. $32.5 \%$ ). Nevethless, further analysis confirmed that ICA positively affects the postoperative outcome independently from ERAS protocol application. Anyway, a minimally invasive approach, irrespective of the anastomotic technique, is recommended to improve the clinical outcome after ERAS application [37].

Lastly, data showed a statistically significant improvement in the ICA group for the postoperative pain control from the 12th $\mathrm{h}$ after surgery with maximum benefits after the 48th in patients not treated with opioids.

\section{Conclusion}

The present Italian multicentric prospective observational study frames the laparoscopic surgeons' attitude about the technical aspects of ileo-colic anastomosis after elective laparoscopic right hemicolectomy for any indications. The skill of the surgeons has allowed the standardization of the totally intracorporeal technique in each high volume laparoscopic center: a side-to-side isoperistaltic stapled ICA with hand-sewn enterotomy closure is the most frequently adopted technique.

No predictive factors for anastomotic complications have been identified among the population characteristics.

The study confirms that intracorporeal anastomosis has advantages in relation to the onset of postoperative complications, especially Clavien-Dindo I-II. The technique by itself has positive influence on the postoperative recovery: it showed better short term outcome with significant reduction of the length of hospital stay, independently from ERAS protocol application.

Finally, these results allow some speculation: the diffusion of the ICA technique is based on better perioperative outcomes and, even more, may be parallel with an increased confidence with advanced laparoscopic colorectal surgery enhanced by vision technologies.

Acknowledgements The study was approved by the Ethical Committee of the province of Ferrara, authorized by the Azienda Ospedaliero-Universitaria of Ferrara with the Protocol No. 170695 and it was registered on clinicaltrial.gov (NCT03934151).

SICE CoDIG (ColonDxItalianGroup): V. Adamo (S Lazzaro Alba - CN), F. Agresta (Adria - RO), A. Agrusa (Palermo), G. Alemanno (Firenze), M.E. Allaix (Torino), A. Alò (Orbassano TO), A. Altamura (Tricase - LE), A. Ambrosi (Foggia), G. Anania (Ferrara), M. Antoniutti (Bassano del Grappa - VI), D. Apa (Roma), G. Arcuri (Gubbio - PG), A. Arezzo (Torino), G.L Baiocchi (Brescia), A. Balani (Gorizia), G. Baldazzi (Milano), M. Basti (Pescara), C. Benvenuto (Protogruaro - VE), C. Bergamini (Firenze), S. Berti (La Spezia), L. Boni (Milano), F. Borghi (Cuneo), E. Botteri (Brescia), R. Brachet Contul (Aosta), A. Brescia (Roma), A. Budassi (Frabriano - AN), L. Cafagna (Andria), M. Calgaro (S Lazzaro Alba - CN), P.G. Calò (Cagliari), R. Campagnacci (Jesi - AN), G. Canova (Borgosesia - VC), G.L. Canu (Cagliari), V. Caracino (Pescara), P. Carcoforo (Ferrara), M. Carlini (Roma), L. Casali (Fidenza - PR), D. Cassetti (Siena), E. Cassinotti (Milano), M. Catarci (Ascoli Piceno), M. Cesari (Città di Castello - PG), P. Checcacci (Firenze), P. Ciano (Ascoli Piceno), M. 
Clementi (L' Aquila), G. Cocorullo (Palermo), F. Colombo (Milano), G. Concone (Milano), A. Contine (Città di Castello - PG), M. Coppola (Lanusei - NU), A. Coratti (Firenze), F. Corcione (Napoli), P. Corleone (Trieste), L. Covotta (Contrada Pozzillo - AV), D. Cuccurullo (Napoli), P. Cumbo (Chieri - TO), G. D'Ambrosio (Roma), F. De Angelis (Latina), M. De Luca (Montebelluna-TV), N. De Manzini (Trieste), C. De Nisco (Nuoro), G.D. De Palma (Napoli), P. De Paolis (Torino), M. Degiuli (Orbassano - TO), D. Delogu (Lanusei - NU), P. Delrio (Napoli), A. Deserra (Cagliari), A. Donini (Perugia), U. Elmore (Milano), G. Ercolani (Forlì), E. Erdas (Cagliari), L. Fabris (Cles TN), G. Ferrari (Milano), G. Feo (Valle Oppio - FE), F. Fidanza (Portogruaro - VE), D. Foschi (Milano), R. Galleano (Pietra Ligure - SV), G. Garulli (Rimini), F. Gatti (Milano), A. Gattolin (Mondovì - CN), S. Gelati (Conegliano Veneto - TV), R. Gelmini (Modena), O. Ghazouani (Pietra Ligure - SV), A. Gioffrè (Roma), S. Gobbi (Olbia), V. Grammatico (Chieri - TO), A. Guariniello (Ravenna), S. Giannessi (Pistoia), M. Guerrieri (Ancona), L. Guerriero (Napoli), G. Gullotta (Palermo), H. Impellizzeri (Peschiera del Garda - VR), M. Izzo (Firenze), E. Jovine (Bologna), G. Lezoche (Ancona), C. Lirusso (Udine), R. Lombardi (Bologna), M. Longoni (Milano), A. Lucchi (Riccione RN), A.P. Luzzi (Genova), P. Marini (Roma), A.G. Marrosu (Sassari), A. Martino (Udine), R. Mazza (Perugia), S. Mazzoccato (Jesi - AN), F. Medas (Cagliari), A. Meloni (San Gavino Monreale - VS), M. Milone (Napoli), E. Minciotti (Gubbio - PG), F. Monari (Riccione - RN), G. Moretto (Peschiera del Garda - VR), I.A. Muttillo (Roma), G. Navarra (Messina), S. Neri (Sassuolo - MO), A. Oldani (Zingonia - BG), S. Olmi (Zingonia - BG), E. Opocher (Milano), E. Osenda (Trieste), R. Ottonello (Muravera - CA), V. Panebianco (Taormina - CT), M. Pavanello (Conegliano Veneto - TV), F. Pecchini (Modena), L. Pellegrino (Cuneo), D. Pennisi (Gorizia), N. Perrotta (Potenza), D. Pertile (Genova), R. Petri (Udine), W. L. Petz (Milano), A. Picchetto (Roma), M. Piccoli (Modena), B. Pirrera (Rimini), A. Pisani Ceretti (Milano), M. Pisano (Muravera - CA), M. Podda (Nuoro), N. Portolani (Brescia), L. Presenti (Olbia), A. Puzziello (Salerno), S. Razzi (Aosta), D. Rega (Napoli), E. Restini (Bari), G. Ricci (Roma), M. Rigamonti (Cles TN), U. Rivolta (Magenta), V. Robustelli (Pistoia), E. Romairone (Genova), R. Rosati (Milano), E. Rosso (Brescia), F. Roviello (Siena), S. Sala (Sassuolo - MO), M. Santarelli (Torino), G. Sarro (Magenta), A. Sartori (Montebelluna-TV), S. Scabini (Genova), F. Scognamillo (Sassari), R. Sechi (San Gavino Monreale - VS), G. Silecchia (Latina), L. Solaini (Forlì), G. Soliani (Ferrara), P. Soliani (Ravenna), E. Soligo (Vercelli), M. Sorrentino (Latisana - UD); G. Spinoglio (Milano), E. Stratta (Genova), A. Taddei (Firenze), G. Talamo (La Spezia), S. Targa (Valle Oppio - FE), N. Tartaglia (Foggia), S. Testa (Vercelli), P. Ubiali (Pordenone), A. Valeri (Firenze), F. Vasta (Taormina - CT), A. Verzelli (Fabriano - AN), N. Vettoretto (Montichiari - BS), R. Vicentini (L'Aquila), G. Viola (Tricase - LE), V. Violi (Fidenza - PR), M. Zago (Borgosesia - VC), L. Zampino (Milano).

Disclosures Gabriele Anania, Ferdinando Agresta, Elena Artioli, Serena Rubino, Giuseppe Resta, Nereo Vettoretto, Wanda Luisa Petz, Carlo Bergamini, Alberto Arezzo, Giorgia Valpiani, Chiara Morotti, Gianfranco Silecchia have no conflicts of interest or financial ties to disclose.

\section{Funding None.}

Open Access This article is distributed under the terms of the Creative Commons Attribution 4.0 International License (http://creativeco mmons.org/licenses/by/4.0/), which permits unrestricted use, distribution, and reproduction in any medium, provided you give appropriate credit to the original author(s) and the source, provide a link to the Creative Commons license, and indicate if changes were made.

\section{References}

1. Rausa E, Kelly ME, Asti E, Aiolfi A, Bonitta G, Bonavina L (2019) Right hemicolectomy: a network meta-analysis comparing open, laparoscopic-assisted, total laparoscopic, and robotic approach. Surg Endosc 33:1020-1032

2. Ohtani H, Tamamori Y, Artimoto Y, Nishiguchi Y, Maeda K, Hirakawa K (2012) A meta-analysis of the short- and long-term results of randomized controlled trials that compared laparoscopy-assisted and open colectomy for colon cancer. J Cancer 3:49-57

3. Lezoche E, Feliciotti F, Paganini AM, Guerrieri M, De Sanctis A, Minervini S, Campagnacci R (2002) Laparoscopic vs. open hemicolectomy for colon cancer. Surg Endosc 16:596-602

4. Tong TK, Law WL (2007) Laparoscopic versus open right hemicolectomy for carcinoma of the colon. JSLS 11:76-80

5. Jian-Cheng T, Wang SS, Bo Z, Jian F, Liang Z (2016) Total laparoscopic right hemicolectomy with 3-step stapled intracorporeal isoperistaltic ileocolic anastomosis for colon cancer. An evaluation of short term outcomes. Medicine 95:48

6. Casciola L, Ceccarelli G, Di Zitti L (2003) Laparoscopic right hemicolectomy with intracorporeal anastomosis. Technical aspects and personal experience. Minerva Chir 58:621-627

7. Vergis AS, Steigerwald SN, Bhojani FD, Sullivan PA, Hardy KM (2015) Laparoscopic right hemicolectomy with intracorporeal versus extracorporeal anastamosis: a comparison of short-term outcomes. Can J Surg 58:63-68

8. Abrisqueta J, Iban N, Luja J, Hernández Q, Parrilla P (2016) Intracorporeal ileocolic anastomosis in patients with laparoscopic right hemicolectomy. Surg Endosc 30:65-72

9. Krouchev R, Tamana S, Nguyen N, Yorke E, Harris D, Sampath S (2018) Total intracorporeal anastomosis for right hemicolectomy: experience from a Canadian center. Am J Surg 215:905-908

10. Sun X, Qiu H, Fei K, Xu L, Lu J, Zhang G, Xiao Y (2017) Comparison of intra-abdominal infection between intracorporeal anastomosis and extracorporeal anastomosis in patients undergoing laparoscopic right hemicolectomy. Zhonghua Wei Chang Wai Ke Za Zhi 20(8):891-895

11. Bollo J, Salas P, Martinez MC, Hernandez P, Rabal A, Carrillo E, Targarona E (2018) Intracorporeal versus extracorporeal anastomosis in right hemicolectomy assisted by laparoscopy: study protocol for a randomized controlled trial. Int J Colorectal Dis 33:1635-1645

12. Milone M, Elmore U, Di Salvo E, Delrio P, Bucci L, Ferulano GP, Napolitano C, Angiolini MR, Bracale U, Clemente M, D’Ambra M, Luglio G, Musella M, Pace U, Rosati R, Milone F (2015) Intracorporeal versus extracorporeal anastomosis. Results from a multicentre comparative study on 512 right-sided colorectal cancers. Surg Endosc 29:2314-2320

13. Anania G, Santini M, Scagliarini L, Marzetti A, Vedana L, Marino S, Gregorio C, Resta G, Cavallesco G (2012) A totally miniinvasive approach for colorectal laparoscopic surgery. World J Gastroenterol 18:3869-3874

14. Carnuccio P, Jimeno J, Pares D (2014) Laparoscopic right colectomy: a systematic review and meta-analysis of observational studies comparing two types of anastomosis. Tech Coloproctol 18:5-12

15. Chang K, Fakhoury M, Barnajian M, Tarta C, Bergamaschi R (2013) Laparoscopic right colon resection with intracorporeal anastomosis. Surg Endosc 27:1730-1736

16. Feroci F, Lenzi E, Garzi A, Vannucchi A, Cantafio S, Scatizzi M (2013) Intracorporeal versus extracorporeal anastomosis after laparoscopic right hemicolectomy for cancer: a systematic review and meta-analysis. Int J Colorectal Dis 28:1177-1186 
17. Lee KH, Ho J, Akmal Y, Nelson R, Pigazzi A (2013) Short- and long-term outcomes of intracorporeal versus extracorporeal ileocolic anastomosis in laparoscopic right hemicolectomy for colon cancer. Surg Endosc 27:1986-1990

18. Magistro C, Di Lernia S, Ferrari G, Zullino A, Mazzola M, De Martini P, De Carli S, Forgione A, Bertoglio CL, Pugliese R (2013) Totally laparoscopic versus laparoscopic-assisted right colectomy for colon cancer: is there any advantage in short-term outcomes? A prospective comparative assessment in our center. Surg Endosc 27:2613-2618

19. ClearyI RK, Kassir A, Johnson CS, Bastawrous AL, Soliman MK, Marx DS, Giordano L, Reidy TJ, Parra-Davila E, Obias VJ, Carmichael JC, Pollock D, Pigazzi A (2018) Intracorporeal versus extracorporeal anastomosis for minimally invasive right colectomy: a multi-center propensity scorematched comparison of outcomes. PLoS ONE 13(10):e0206277

20. Ricci C, Casadei R, Alagna V, Zani E, Taffurelli G, Pacilio CA, Minni F (2017) A critical and comprehensive systematic review and meta-analysis of studies comparing intracorporeal and extracorporeal anastomosis in laparoscopic right hemicolectomy. Langenbecks Arch Surg 402:417-427

21. Scatizzi M, Kroning KC, Borrelli A, Andan G, Lenzi E, Feroci F (2010) Extracorporeal versus intracorporeal anastomosis after laparoscopic right colectomy for cancer: a case-control study. World J Surg 34:2902-2908

22. Tarta C, Bishawi M, Bergamaschi R (2013) Intracorporeal ileocolic anastomosis: a review. Tech Coloproctol 17:479-485

23. van Oostendorp S, Elfrink A, Borstlap W, Schoonmade L, Sietses C, Meijerink J, Tuynman J (2017) Intracorporeal versus extracorporeal anastomosis in right hemicolectomy: a systematic review and meta-analysis. Surg Endosc 31:64-77

24. Shapiro R, Keler U, Segev L, Sarna S, Hatib K, Hazzan D (2016) Laparoscopic right hemicolectomy with intracorporeal anastomosis: short- and long-term benefits in comparison with extracorporeal anastomosis. Surg Endosc 30:3823-3829

25. Arezzo A, Passera R, Ferri V, Gonella F, Cirocchi R, Morino M (2015) Laparoscopic right colectomy reduces short-term mortality and morbidity. Results of a systemic review and meta-analysis. Int J Colorectal Dis 30:1457-1472

26. Cirocchi R, Trastulli S, Farinella E, Guarino S, Desiderio J, Boselli C, Parisi A, Noya G, Slim K (2013) Intracorporeal versus extracorporeal anastomosis during laparoscopic right hemicolectomy-systemic review and meta-analysis. Surg Oncol 22:1-13

27. Biondi A, Santocchi P, Pennestri F, Santullo F, D'Ugo D, Persiani R (2017) Totally Laparoscopic right colectomy versus laparoscopically assisted right colectomy: a propensity score analysis. Surg Endosc 31:5257-5282

28. Horan AC, Andrus M, Dudeck MA (2008) CDC/NHSN surveillance definition of health care-associated infection and criteria for specific types of infections in the acute care setting. Am J Infect Control 36(5):309-332

29. Clavien PA, Barkum J, de Oliveira ML, Vauthey JN, Dindo D, Schulick RD, de Santibañes E, Pekolj J, Slankamenac K, Bassi
C, Graf R, Vonlanthen R, Padbury R, Cameron JL, Makuuchi M (2009) The Clavien-Dindo classification of surgical complication: 5-years experience. Ann Surg 250(2):187-196

30. Martinek L, You K, Giuratrabocchetta S, Gachabayov M, Lee K, Bergamaschi R (2018) Does laparoscopic intracorporeal ileocolic anastomosis decreases surgical site infection rate? A propensity score-matched cohort study. Int J Colorectal Dis 33:291-298

31. Milone M, Elmore U, Vignali A, Gennarelli N, Manigrasso M, Burati M, Milone F, De Palma GD, Delrio P, Rosati R et al (2018) Recovery after intracorporeal anastomosis in laparoscopic right hemicolectomy: a systematic review and meta-analysis. Langenbeck's Arch Surg 403:1-1

32. Hanna MH, Hwang GS, Phelan MJ, Bui TL, Carmichael JC, Mills SD, Stamos MJ, Pigazzi A (2016) Laparoscopic right hemicolectomy: short- and long-term outcomes of intracorporeal versus extracorporeal anastomosis. Surg Endosc 30:3933-3942

33. Elmore U, Vignali A, Rosati R, Valeri A, Silecchia G, SICE Colorectal Collaborative Group (2019) SICE national Survey: current state on the adoption of laparoscopic approach to the treatment of colorectal disease in Italy. Update Surg 43(2):201-207

34. Frasson M, Granero-Castro P, Ramos Rodríguez JL, Flor-Lorente B, Braithwaite M, Martí Martínez E, Álvarez Pérez JA, Cazador A, Espí A, Garcia-Granero E, ANACO Study Group et al (2015) Risk factors for anastomotic leak and postoperative morbidity and mortality after elective right colectomy for cancer: results from a prospective, multicentric study of 1102 patients. Int J Colorectal Dis 31:105-114

35. Aquina CT, Mohile SG, Tejani MA, Becerra AZ, Xu Z, Hensley BJ, Arsalani-Zadeh R, Boscoe FP, Schymura MJ, Noyes K, Monson JR, Fleming FJ (2017) The impact of age on complications, survival, and cause of death following colon cancer surgery. Br J Cancer 116:389-397

36. The 2015 European Society of Coloproctology Collaborating Group (2017) Relationship between method of anastomosis and anastomotic failure after right hemicolectomy and ileocaecal resection: an international snapshot audit. Colorectal Dis 19:296-311

37. Gustafsson UO, Scott MJ, Hubner M, Nygren J, Demartines N, Francis N, Rockall TA, Young-Fadok TM, Hill AG, Soop M, de Boer HD, Urman RD, Chang GJ, Fichera A, Kessler H, Grass F, Whang EE, Fawcett WJ, Carli F, Lobo DN, Rollins KE, Balfour A, Baldini G, Riedel B, Ljungqvist O (2019) Guidelines for preoperative care in elective colorectal surgery: enhanced recovery after surgery (ERAS) Society Recommendation: 2018. World J Surg 43(3):659-695

Publisher's Note Springer Nature remains neutral with regard to jurisdictional claims in published maps and institutional affiliations.

\section{Affiliations}

\section{Gabriele Anania $^{1,2}$ - Ferdinando Agresta ${ }^{3}$ - Elena Artioli ${ }^{1,2}$. Serena Rubino ${ }^{1,2}$. Giuseppe Resta ${ }^{1,2}$. Nereo Vettoretto ${ }^{4}$. Wanda Luisa Petz ${ }^{5}$. Carlo Bergamini ${ }^{6} \cdot$ Alberto Arezzo $^{7}$. Giorgia Valpiani ${ }^{8}$. Chiara Morotti ${ }^{8}$. Gianfranco Silecchia ${ }^{9}$. SICE CoDIG (Colon Dx Italian Group)}

1 Division of General Surgery, S. Anna University Hospital of Ferrara, via Aldo Moro 8, Cona, FE, Italy
2 Department of Morphology, Surgery and Experimental Medicine, University of Ferrara, Ferrara, Italy 
3 Department of General Surgery, ULSS5 Polesana del Veneto, Adria, RO, Italy

4 Montichiari Surgery, ASST Spedali Civili Brescia, Montichiari, BS, Italy

5 Division of Gastrointestinal Surgery, IEO, European Institute of Oncology IRCCS, Milan, Italy

6 Department of Emergency Surgery, University Hospital of Careggi, Florence, Italy
7 Department of Surgical Sciences, University of Torino, Turin, Italy

$8 \mathrm{MsC}$ in Statistics at Research Innovation Office, S. Anna University Hospital of Ferrara, Ferrara, Italy

9 Department of Medical Surgical Science and Biotechnologies, Faculty Pharmacy and Medicine, Sapienza University of Rome, Rome, Italy 